\title{
Bacterial Vaginosis among Symptomatic Women of Reproductive Age Group in and around NIMS Hospital, Jaipur, India
}

\author{
Shilpa Pradhan ${ }^{1 *}$, Surabhi Tomar ${ }^{2}$, Anjali Kulshrestha ${ }^{1}$, Farkanda $^{3}$ and Suman Rishi $^{3}$ \\ ${ }^{1}$ Department of Microbiology, NIMS Medical College, Shobha Nagar, Jaipur, India \\ ${ }^{2}$ Department of Obstetrics and Gynaecology and IVF division, NIMS Hospital, \\ Shobhanagar, Jaipur, India \\ ${ }^{3}$ Department of Microbiology, NIMS Medical College, Shobha Nagar, Jaipur, India \\ *Corresponding author
}

\section{A B S T R A C T}

Bacterial vaginosis (BV) is a polymicrobial, most common cause of abnormal vaginal discharge among young women of Reproductive age groups. This cross sectional study were to analyze the prevalence of vaginal infections in the patients attending Obstetrics

\begin{tabular}{|c|}
\hline $\begin{array}{l}\text { Ke y w o r d s } \\
\text { BV, Gram stain, } \\
\text { Culture, Wet } \\
\text { mount. }\end{array}$ \\
\hline Article Info \\
\hline $\begin{array}{l}\text { Accepted: } \\
\text { 19 June } 2017 \\
\text { Available Online: } \\
10 \text { August } 2017\end{array}$ \\
\hline
\end{tabular}
and Gynaecology with various complaints like increased malodorous vaginal discharge among sexually active women aged 15-45yrs. We processed 100 High Vaginal Swabs (HVS) of Symptomatic women attending our OPD's between January and June 2017(06 months). Each HVS were subjected to Wet mount, Gram stain and Culture on BA, Mac Conkey Agar, SDA. Wet mounts were done to identify Trichomonas vaginalis and Gram stains were done to look for bacterial morphotypes. A total of 100 women participated in the study with the overall prevalence of BV rated $39 \%$ out of 39 samples 12 samples grew MS CoNS (30.76\%), 08 samples grew MS CoPS (20.51\%),07 grew E. coli (17.94\%), 04 grew Enterococcus spp (10.25\%) and 08 samples yielded anaerobic bacteria (20.51\%). $21 \%$ were suffering from VVC. Out of these $21 \%$ of Candida spp- 10 Samples grew Candida albicans (47\%) and 11 samples yielded Non-albicans Candida (52.4\%). 05 samples also yielded Trichomonas vaginalis in the wet mounts. We got a wide spectrum of bacteria of $39 \%$ and fungi of $21 \%$; $05 \%$ also showed Trichomonal infection. Overall $60 \%$ of the symptomatic women showed microbiological correlation.

\section{Introduction}

Bacterial vaginosis (BV) is a disease of the vagina caused by excessive growth of the bacteria (Donders et al., 2014; Clark et al., 2014). BV caused by an imbalance of the naturally occurring bacteria in the vagina (Bennett, 2015; Bacterial Vaginosis (BV), 2015). Here there is reduction in the amount of hydrogen peroxide producing Lactobacilli and overgrowth of anaerobic bacteria especially Mycoplasma hominis, Bacteriodes spp, Mobiluncus spp and Gardnerella vaginalis (Akomoneh et al., 2016). Bacterial vaginosis is an extremely prevalent condition and the number one cause of vaginitis among sexually active women (Yudin and Money, 2008).

Although it is not a reportable disease, available data show the prevalence of $\mathrm{BV}$ among non-pregnant women to range from 
15-30\% and 50\% for pregnant women (Fleury et al., 1981; Shayo et al., 2012). However, the majority of cases of BV are asymptomatic and remain unreported and untreated (Amsel et al., 1983).

BV presents with fishy vaginal discharge usually white or grey in color. Burning with micturation may occur (What are the symptoms of Bacterial Vaginosis, 2015). Risk factors for BV include sexual activity, new or multiple sexual partners and early age of sexual intercourse. Bacterial vaginosis is a risk factor for acquisition of herpes simplex virus type 2, gonorrhoea and chlamydial infections (Cherpes et al., 2003). The incidence of $\mathrm{BV}$ has also been associated with a greater occurrence of other sexually transmitted infections like HIV and cytomegalovirus (Joesoef et al., 1995) (Prevalence and correlates of Bacterial vaginosis among young women of Reproductive age in Mysore, India).

Previously considered a benign condition, BV has been implicated in many gynaecologic conditions and complications of pregnancy including Pelvic inflammatory disease, post hysterectomy vaginal cuff cellulites, endometriosis, amniotic-fluid infection, preterm delivery, pre-term labour, pre-mature rupture of membranes and possibly spontaneous abortions (Eschenback et al., 1988). BV resolves spontaneously in up to one third of non-pregnant and one -half of pregnant women (Klebanoff et al., 2004). Treatment is indicated for relief of symptoms in women with symptomatic infection and to prevent post operative infection in those with symptomatic infection prior to abortion or hysterectomy or any post vaginal surgical procedure. Treatment of BV may also reduce the risk of acquiring sexually transmitted diseases (STD's), including HIV (Schwebke et al., 2004). BV is of special public health concern in India because of the high burden of reproductive and pregnancy -related morbidity. Research in India is sparse and mainly limited to a few states (Prevalence and correlates of Bacterial vaginosis among young women of Reproductive age in Mysore, India). To date, there are only few studies on the prevalence of $\mathrm{BV}$ in the India.

Vulvovaginitis is a common day today problem in gynaecologic practice. Candida albicans is the commonest infectious cause producing symptoms and signs of vulvovaginal pruritis, burning irritation, soreness, burning of micturation, dysparunia and whitish cheesy discharge (Cheesbrough, 2006). However, a shift towards Non-albicans Candida spp has been recently observed. These Non-albicans Candida spp demonstrate reduced susceptibility to commonly used antifungal drugs.

So the current cross sectional study was carried out to know the microbiological profile of symptomatic women attending the Gynaecological OPD's in NIMS Hospital, Shobha nagar, Jaipur so as to provide proper treatment in order prevent future complications occurring because of them.

\section{Materials and Methods}

NIMS Medical College and Research Centre is a 950 bedded hospital located in Jaipur with an average of 50-60 patients in Gynaecology OPD each day. In this cross sectional study we selected 100 women attending Gynaecology OPD with symptoms of BV. All the samples were collected with strict aseptic precautions using sterile swabs. And the swabs were sent to the Department of Microbiology immediately after collection.

After the required entries, the swabs were first subjected to wet mount preparation, gram stain and cultures were being put-up on BA, Mac Conkey Agar and SDA. Wet mount preparation was done to identify Tricomonas vaginalis (motile). Gram stains were done to 
identify Gram variable bacteria with different morphotypes and CLUE cells. Nugent's scoring system was applied to grade the smears. Cultures were inspected on the subsequent day for the growth and necessary biochemical reactions were done to identify them. Antibiotic susceptibility testing was carried out using Kirby Bauer method. Growths on SDA were subjected to Lacto phenol cotton blue preparation to identify the fungal morphotypes. Growths showing Candida spp were further subjected to Germ tube tests and sub cultured on CHROM Agar to identify the species, Corn meal agar to look for Chlamydospores. Fermentation and Assimilation tests were carried out where ever required. Anti-fungal susceptibility was carried out in case of Candida spp only.

\section{Observation}

Total of 100 Samples were processed. Each sample was subjected to Wet mount preparation to look for Trichomonas vaginalis.

\section{SALINE WET MOUNT}

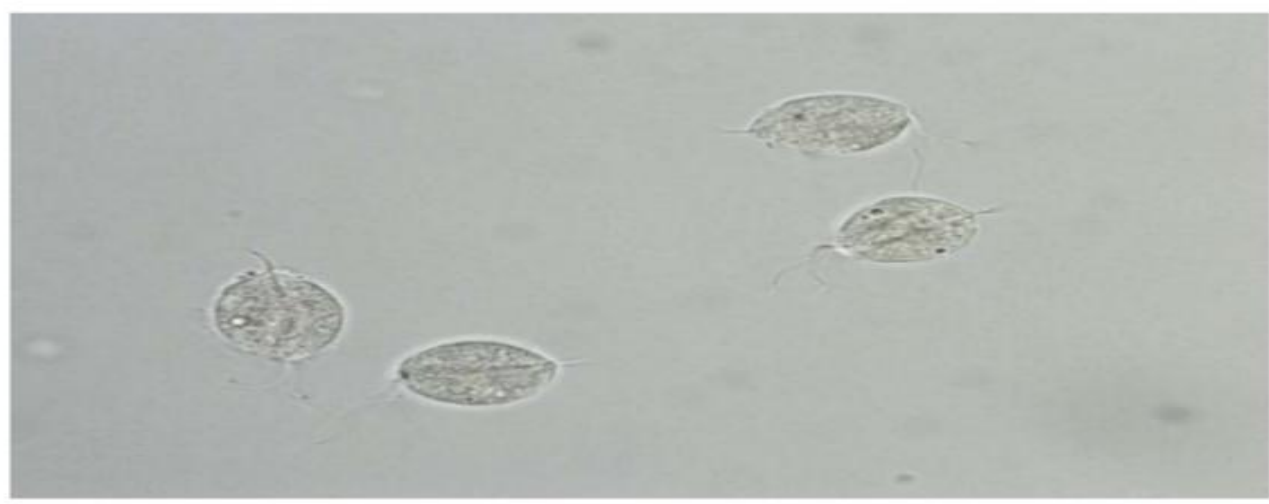

Wet mount preparation

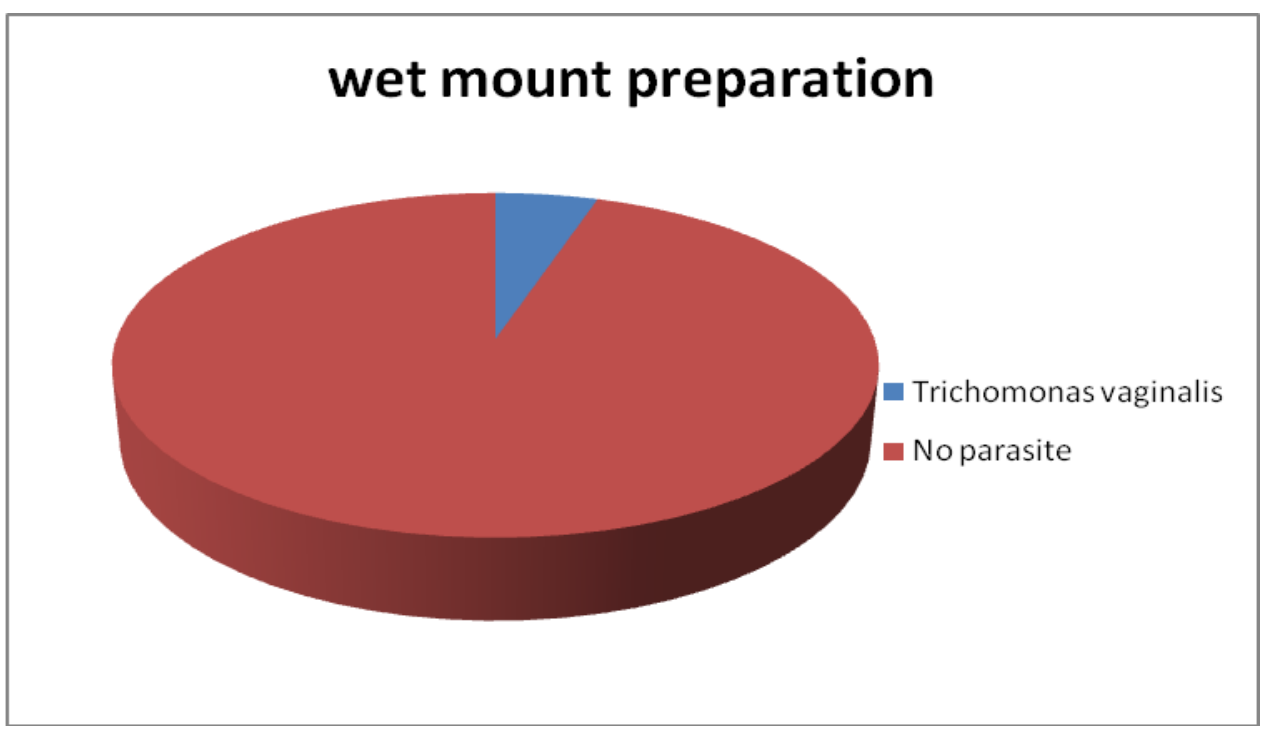




\section{Gram stain showing Candida species}

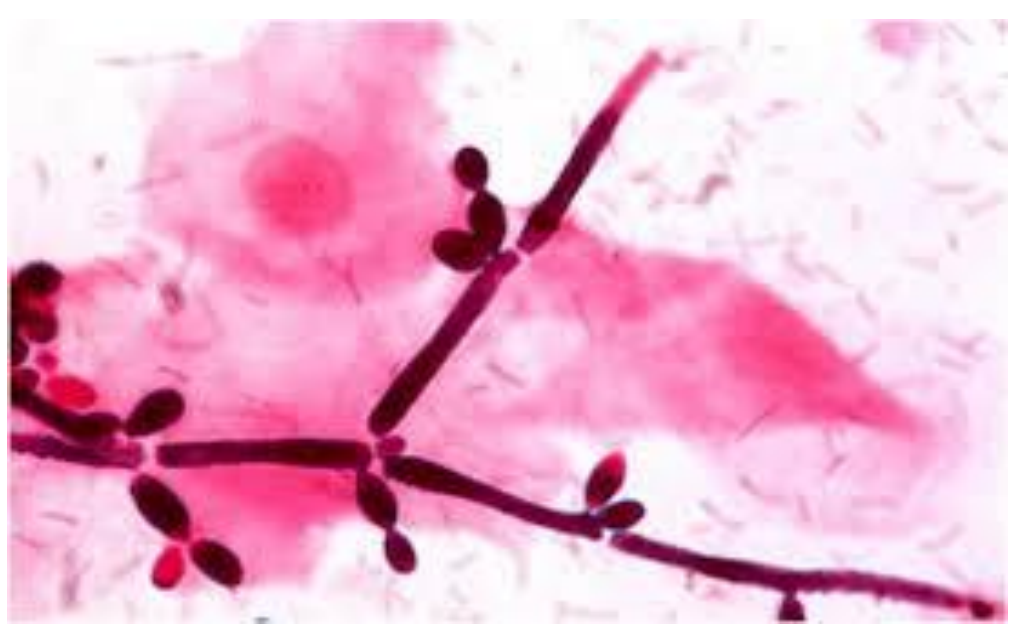

Gram stain showing Lactobacilli

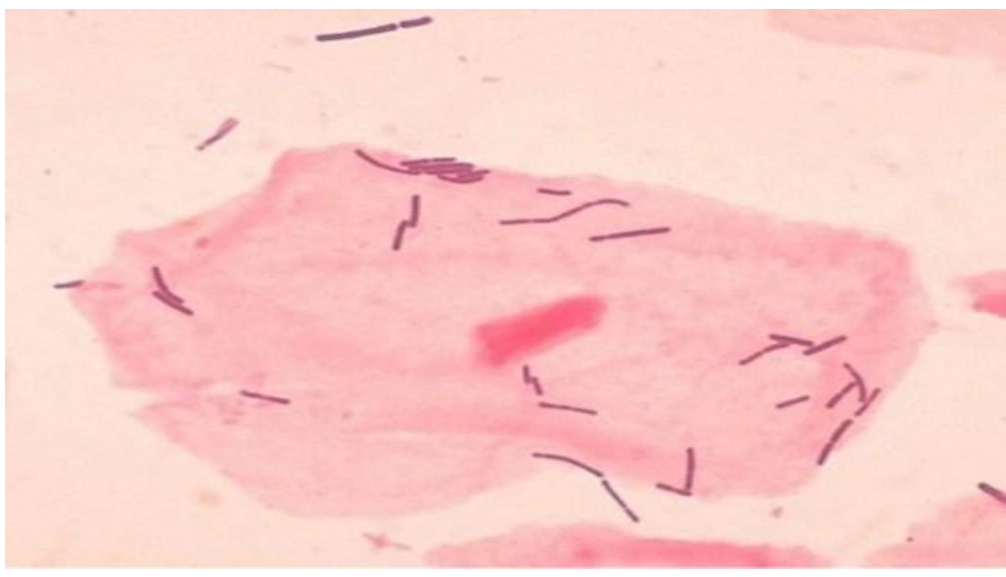

CLUE cells

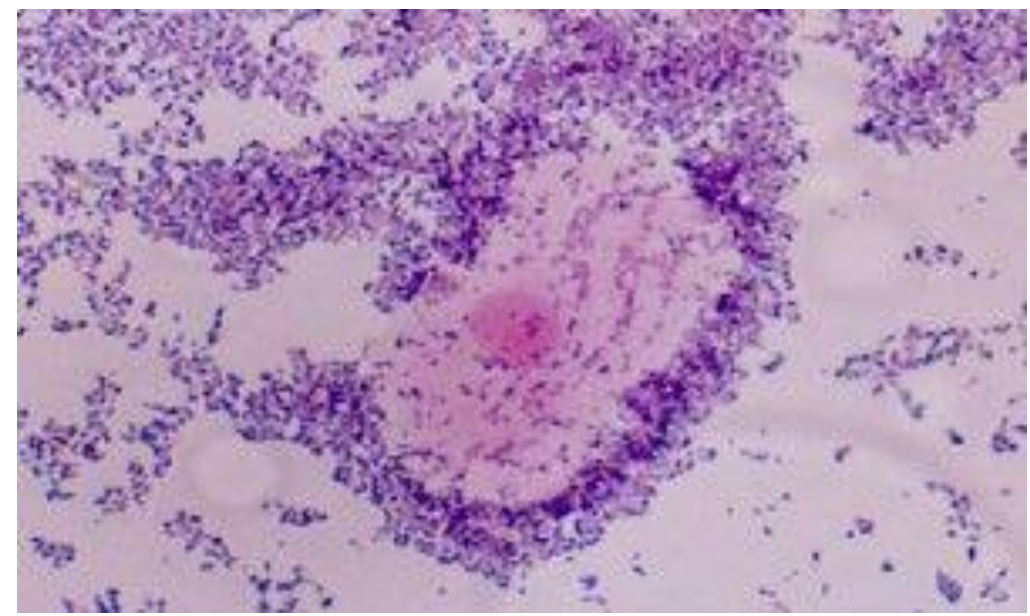


Mac Conkey showing $E$. coli

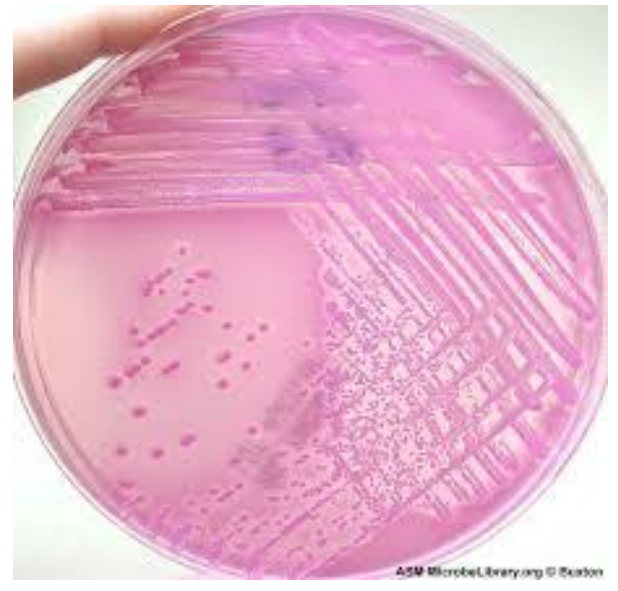

BA showing Staphylococcus aureus

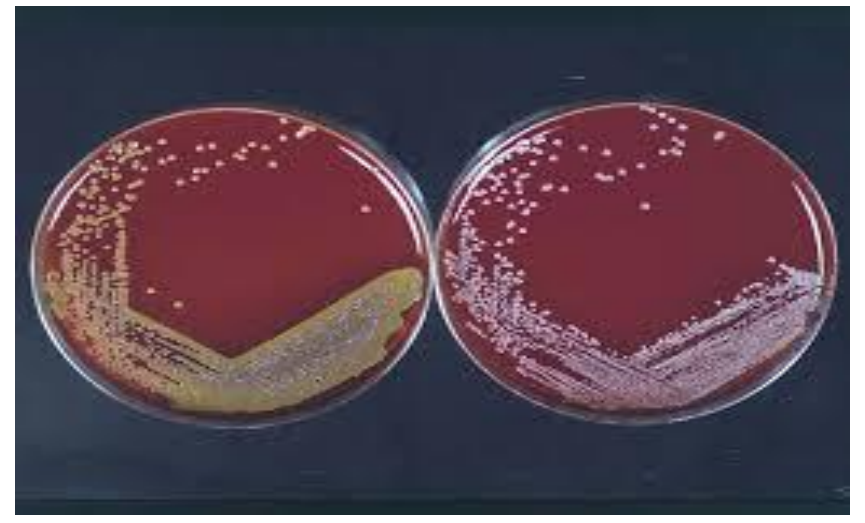

Antibiotic susceptibility testing done by Kirby Bauer method

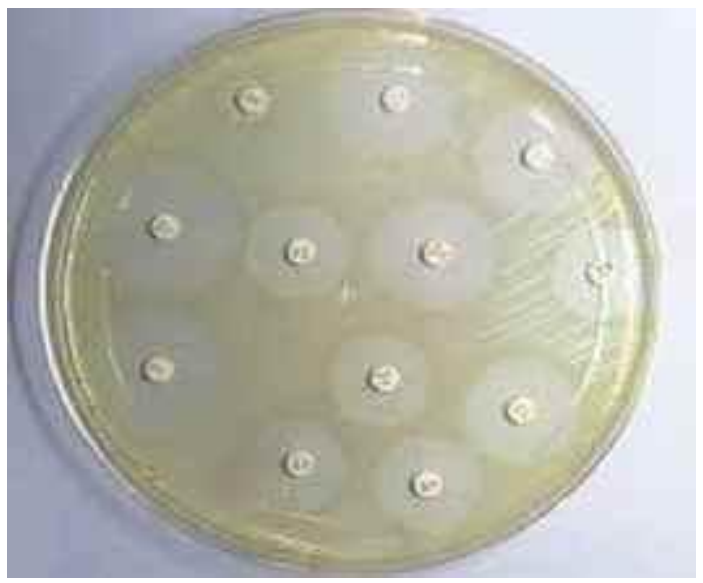




\section{BA showing Candida spp}

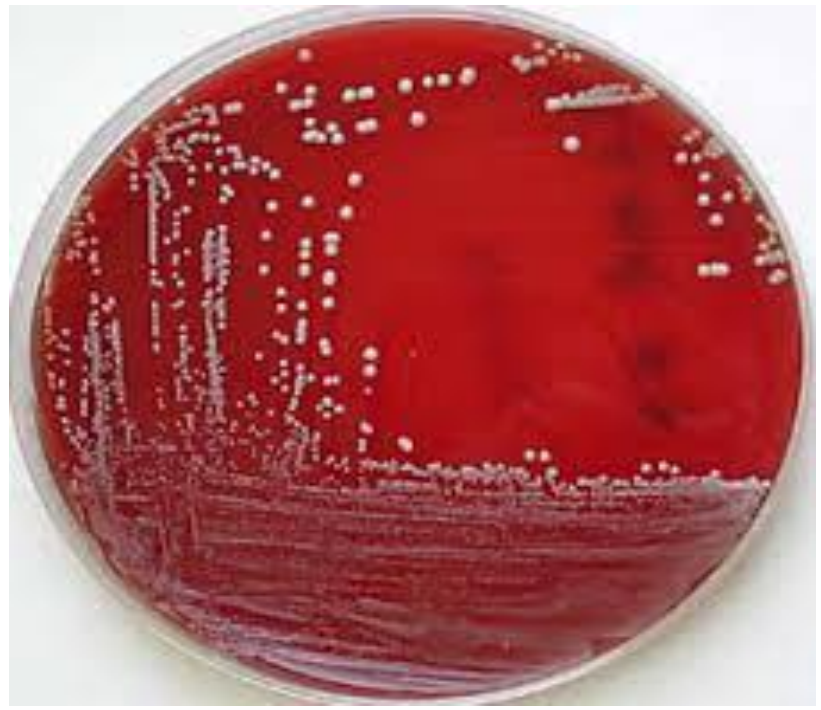

SDA slant showing subculture of Candida spp

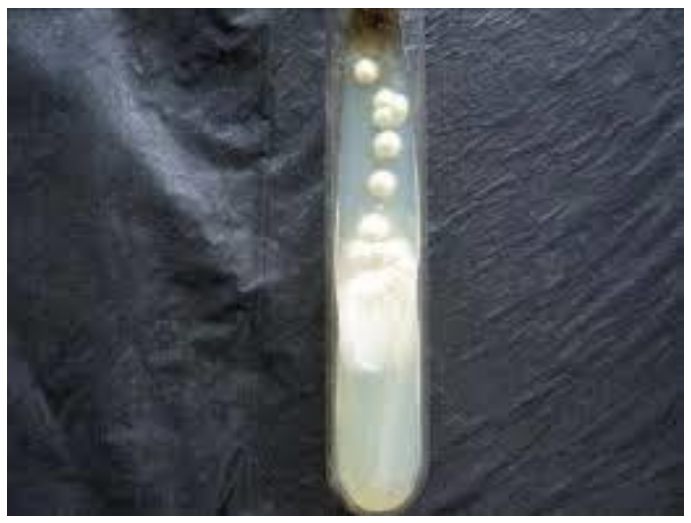

Chrom agar showing Candida albicans

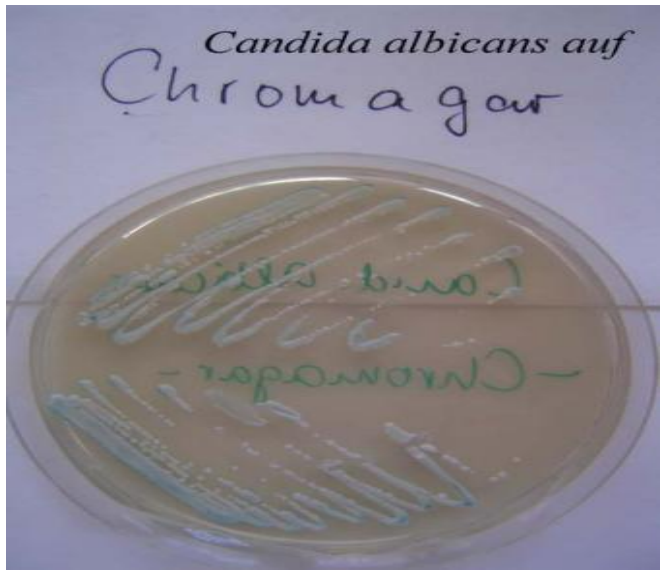

1790 
Total isolated organisms

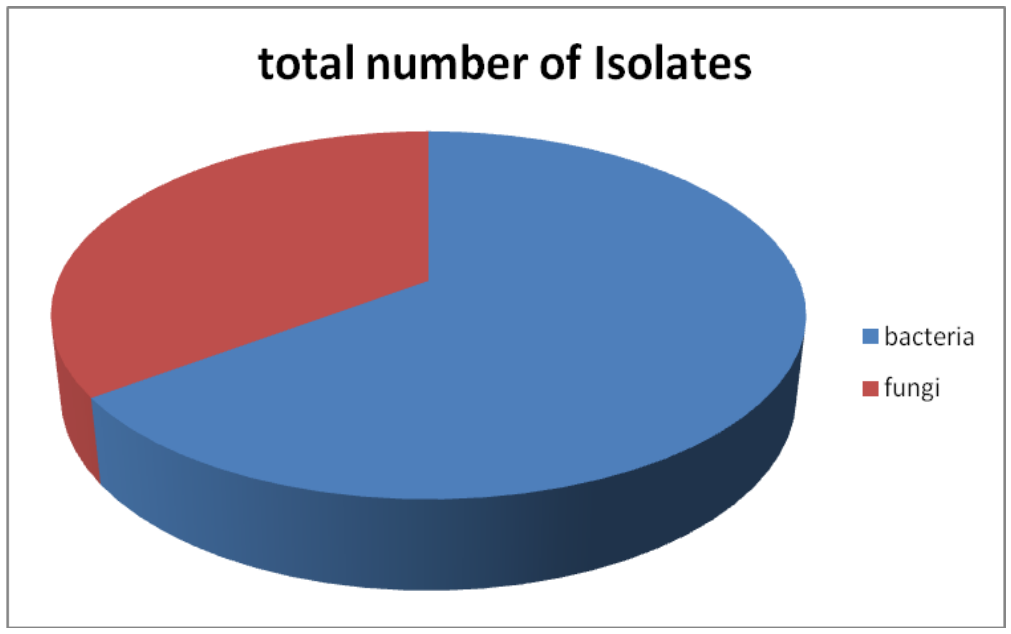

Total number of bacterial isolates

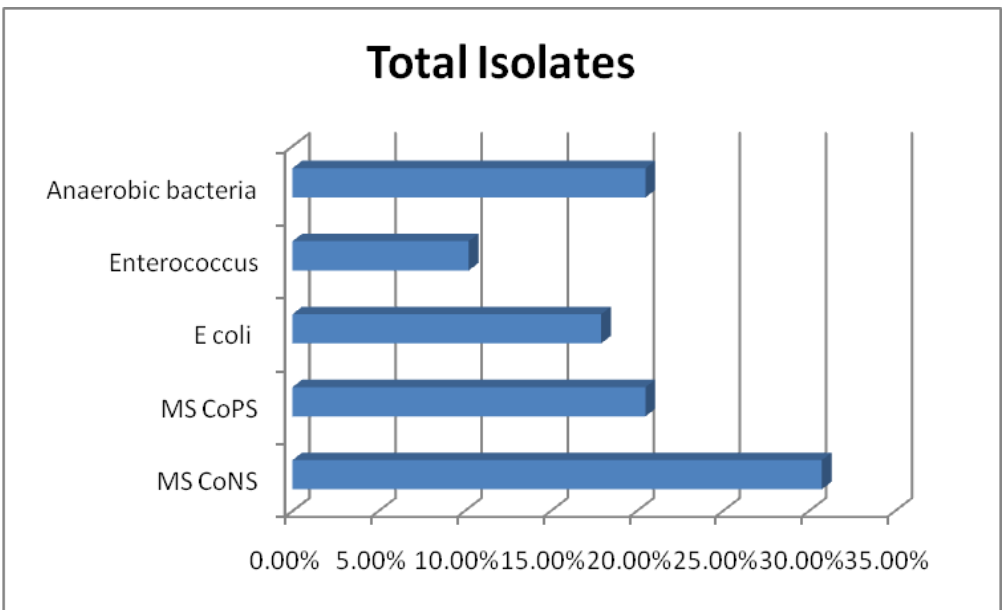

Distribution of fungal isolates

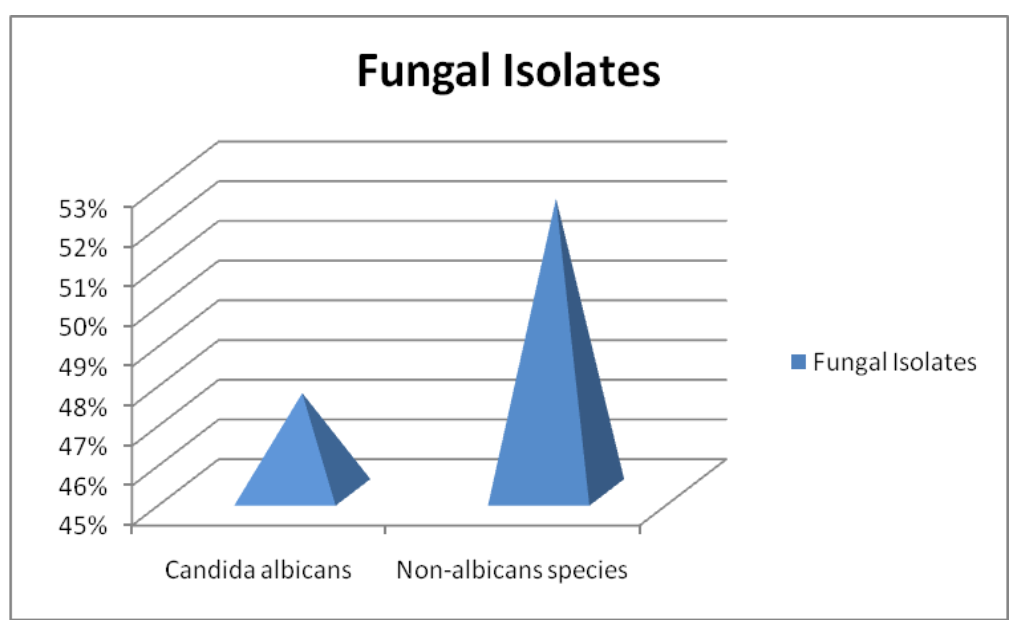




\section{Distribution of Non-albicans species isolated}

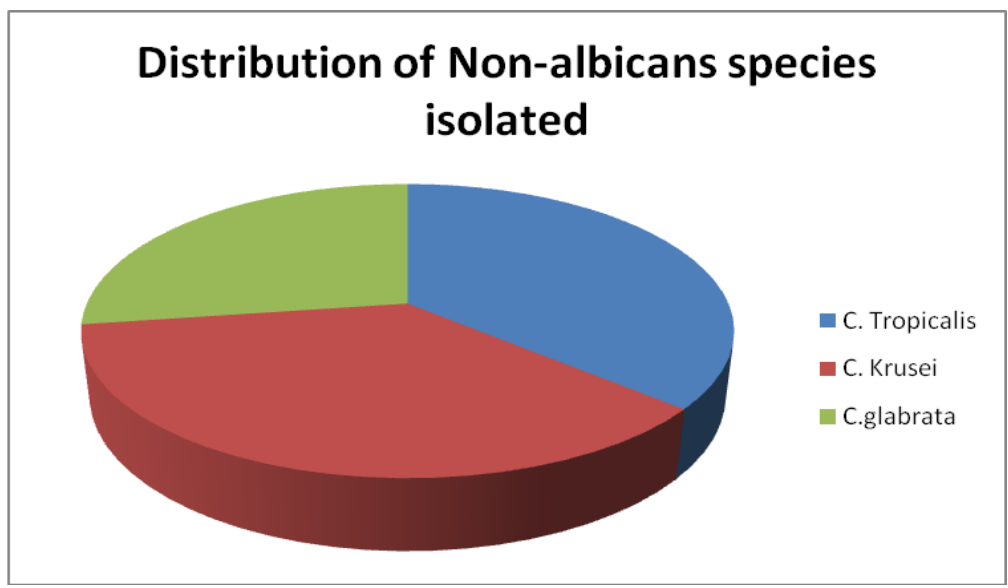

\section{Results and Discussion}

The current study was done to the Prevalence of BV in the patients attending Obgy OPD at NIMS Hospital, Jaipur between January and June 2017. A total of 100 Symptomatic women were included in this cross sectional study. Out of 100 women, the overall prevalence of BV was $39 \%$ out of 39 samples 12 samples grew MS CoNS (30.76\%), 08 samples grew MS CoPS (20.51\%),07 grew E coli(17.94\%), 04 grew Enterococcus spp $(10.25 \%)$ and 08 samples yielded anaerobic bacteria $(20.51 \%)$.

As much as $21 \%$ were suffering from VVC. Out of these $21 \%$ of Candida spp- 10 Samples grew Candida albicans (47\%) and 11 samples yielded Non-albicans Candida (52.4\%). Among Non-Candida spp 04 cultures showed to be Candida tropicalis (36.36) and Candida krusei (36.36), 03(27.27) cultures were that of Candida glabrata. We also noticed 05 samples showing Trichomonas vaginalis in the wet mounts.

We got a wide spectrum of bacteria of $39 \%$ and fungi of $21 \%$. Our study showed relatively high prevalence rate of $\mathrm{BV}$. The reasons may be because of poverty, lack of literacy and lack of personal hygiene knowledge and rural patients attending the
OPD. We compared our study with a study group conducted at Mysore and Cameroon. They correlated well with our BV prevalence rates. Similarly we compared our Candidal infection rates to a study conducted at Govt Medical College, Amritsar, which also suggested similar clinical culture positivity.

We also noticed that the Prevalence of Nonalbicans Candida species is increasing. This shift from Candida albicans to Non-albicans Candida is alarming, as these Non-albicans Candida spp show reduced susceptibility to commonly used anti-fungal drugs.

Treatment is recommended only for women with symptoms. The other potential benefit of the treatment is reduction in acquiring C. trachomatis, N. gonorrhoea, T. vaginalis, HIV and Herpes Simplex type 2. Usually Metronidazole is sufficient for oral or intravaginal gel. Clindamycin can also be the alternate drug of choice. Oral Anti-fungals can be given for VVC along with topical antifungal pessaries, vaginal tablets or creams. Overall $60 \%$ of the symptomatic women showed microbiological correlation.

\section{References}

Akomoneh Elvis Achondou, Foche Francis Fumoloh, Aseneck Christian Aseneck, 
Abong Ralph Awah and Ajonina Marcelus Utokoro. 2016. Afr. J. Infect. Dis., 10(2): 96-10 Published online 2016, doi:10.2010/ajid.v10:2.4 PMCID: PMCJ 411994.

Bacterial Vaginosis (BV). 2015. Condition Information, National Institute of Child Health and Human Development. Prevalence of Bacterial Vaginosis among Sexually active women attending the CDC Cetral Clinic TIKO, South West Region, Cameroon.

Bennett, John. 2015. Mandell, Douglas, and Bennett's principles and practice of infectious diseases. Philadelphia, PA: Elsevier/ Saunders.

Cheesbrough, M. 2006. Microbiological tests; chapter 7, District Laboratory Practice in Tropical Countries, $\mathrm{Pt} 2.2^{\text {nd }}$. Cambridge: Cambridge University Press, pp. 94-96.

Clark, Natalie, Tal, Reshef, Sharma, Harsha, Segars, James. 2014. "Microbiota and Pelvic Inflammatory Disease." Seminars in Reproductive Med.,
32(01):043-049. Doi:10. 1055/s-00331361822.

Donders, G.G., Zodzika, J., Rezeberge, D. 2014. "Treatment of Bacterial vaginosis: what we have and what we miss. Expert Opinion on Pharmacotherapy, 15(5): 645-657. Doi: 10.1517/14656566. 2014.881800.

Jindal Neerja, Aggarwal Aruna, Gill Paramjeet. 2006. Significance of Candida Culture in Women with Vulvovaginal symptoms. J. Obstet. Gynecol. India, Vol 56 No 2, P. 139141.

Madhivanan, K. Krupp, V. Chandrasekaran, C. Karat, C.R. Cohen, A.L. Reingold and J.D. Klausner. Indian J. Med. Microbiol., 26(2): 132-137.

Prevalence and correlates of Bacterial vaginosis among young women of Reproductive age in Mysore, India.

What are the symptoms of Bacterial Vaginosis? 2013-05-21. Retrieved 3 March 2015.

\section{How to cite this article:}

Shilpa Pradhan, Surabhi Tomar, Anjali Kulshrestha, Farkanda and Suman Rishi. 2017. Bacterial Vaginosis among Symptomatic Women of Reproductive Age Group in and around NIMS Hospital, Jaipur, India. Int.J.Curr.Microbiol.App.Sci. 6(8): 1785-1793. doi: https://doi.org/10.20546/ijcmas.2017.608.211 